\title{
Investigations on the general properties of biomass-based aldehyde tanned sheep fur for its selective post-tanning processing
}

\author{
Wei Ding ${ }^{*^{*}}$, Ya-nan Wang ${ }^{2,3}$, Jianfei Zhou ${ }^{2,3}$, Haiteng Liu', Xiaoyan Pang ${ }^{1}$ and Bi Shi ${ }^{2,3^{*}}$
}

\begin{abstract}
Dialdehyde sodium alginate (DSA) is an alternative chrome-free tanning material for fur production. To obtain satisfactory resultant fur and provide suggestions for the usage of DSA in fur making, the general properties of DSA tanned sheep fur were systematically investigated. The tanning mechanism of DSA was analyzed and it was verified that DSA was mainly combined with collagen fiber by forming Schiff base covalent bonds while supplemented by a small number of hydrogen bonds and ionic bonds. Due to the acid sensitivity of Schiff base structure, DSA tanned fur had poor resistance to acid rinsing but had excellent resistance to washing and good fatliquoring performance. Also, it had good resistances to yellowing and reductant. After being retanned by chrome tanning agent, the fur was capable of enduring a high-temperature dyeing process $\left(68^{\circ} \mathrm{C}\right.$ for $\left.8 \mathrm{~h}\right)$. Overall, DSA tanned sheep fur had favorable properties under appropriate post-tanning processing conditions to manufacture lightcolored or dark-colored fur products with desirable physical properties.
\end{abstract}

Keywords: Sheep fur, Dialdehyde sodium alginate, Stability, Post-tanning, Chrome retanning

\section{Introduction}

Sheep fur manufacturing is a sustainable industry since it converts raw sheep fur, the byproduct of meat industry, into high value-added products [1]. Sheep fur production generally involves numerous processes. Among them, tanning process is the key one since it determines the hydrothermal stability, dispersion of collagen fiber, and the charge property of tanned fur. These properties will affect the stability of fur under posttanning processing conditions as well as the uptake and fixation of post-tanning chemicals [2, 3]. Post-tanning processes, including neutralizing, bleaching, retanning, filling, fatliquoring, dyeing et al., are closely related to the physical and organoleptic properties of resultant fur. At present, chrome tanning is the dominant tannage for

\footnotetext{
* Correspondence: dingwei1368@outlook.com; shibi@scu.edu.cn

${ }^{1}$ China Leather and Footwear Research Institute Co. Ltd., Beijing 100015, P.R.

China

${ }^{2}$ College of Biomass Science and Engineering, Sichuan University, Chengdu 610065, China

Full list of author information is available at the end of the article
}

sheep fur production because it can endow tanned fur with excellent tanning effect and post-tanning processing properties. However, this conventional tannage is suffering from increasing restriction due to the widespread chrome pollution that is hindering the sustainable development of fur industry [2]. In recent years, new chrome-free tanning agents and clean tanning technologies have been developed to partially replace conventional chrome tannage, such as syntans, biomassderived aldehyde and chrome-reduced combination tanning technology $[4,5]$. As a kind of green and sustainable tanning material, the biomass-derived aldehyde is now arousing more and more interest due to its feasible tanning effect and biodegradability [6-9]. Its industrial use will be significant for the effective control of chrome pollution and the sustainable development of fur industry.

Dialdehyde sodium alginate (DSA) is a sort of special biomass-derived aldehyde with multiple carboxyl groups. It is prepared by periodate oxidation of sodium alginate 
(SA) which is composed of (1-4)- $\beta$-D-mannuronic acid (M) units and $\alpha$-L-guluronic acid (G) units $[10,11]$. During the periodate oxidation, the vicinal diols of SA are selectively oxidized to introduce aldehyde groups onto the backbone of DSA along with the decrease of molecular weight $[12,13]$. These structural properties enhance the interactions of DSA with the amino groups of collagen/gelatin molecules and the penetrability in collagen fiber matrix [14-16]. This suggests the possibility of DSA as a tanning agent for fur processing. Recently, we developed a kind of DSA with wide molecular weight distribution, which could exhibit a satisfactory tanning effect on sheep fur in terms of high shrinkage temperature (Ts) and well- dispersed collagen fiber network [1]. This DSA tanned fur exhibited the potential to be processed as light-colored fur products. However, the current post-tanning technologies are designed to match up with chrome tanning system and may be not suitable for DSA tannage in consideration of the low isoelectric point of DSA tanned wet-white (less than 5.0) [17]. In addition, as for the production of dark-colored fur, hightemperature dyeing (about $70^{\circ} \mathrm{C}, 6 \sim 8 \mathrm{~h}$ ) is commonly required for the production of dark-colored sheep fur. The Ts of tanned fur is required to be no lower than $90{ }^{\circ} \mathrm{C}$ for preventing fur from shrinkage during hightemperature dyeing $[4,18]$. This shows that the DSA tanned fur is not qualified when singly used, and it seems that chrome is essential for making dark-colored sheep fur. Considering that DSA has abundant carboxyl groups to coordinate with $\mathrm{Cr}$ (III), chrome-reduced combination tanning based on DSA is a potential approach for dark-colored sheep fur production. However, the current research works about DSA tanning agent mainly focus on its preparation and the relationships between its structure and tanning effect. The general properties of DSA tanned fur under post-tanning processing conditions still remain unclear. In addition, the chromereduced combination tanning scheme based on DSA is difficult to be reasonably proposed due to the unclarified interactions between DSA and Cr (III). Thus, it is significant to clarify the general properties of DSA tanned fur under post-tanning processing conditions for the reasonable use of DSA in fur production.

In the present work, the tanning mechanism of DSA was first analyzed and clarified. According to the tanning mechanism and post-tanning processing conditions, the stabilities of DSA tanned fur, e.g., resistances to acid, washing, reductants, yellowing, and the fatliquoring performance were specifically evaluated. Moreover, the interactions between DSA and chrome tanning agents as well as the chrome-reduced combination tanning performances based on DSA were also investigated. Clarification of the general properties of DSA tanned sheep fur under post-tanning processing conditions is expected to guide the reasonable use of DSA for making satisfactory resultant fur.

\section{Experimental \\ 2.1 Materials}

Pickled sheep fur was provided by Zhejiang Zhonghui Fur \& Leather Co., Ltd. (Tongxiang, China). Dialdehyde sodium alginate was prepared according to the method reported in our previous work [15]. Pickled sheep fur was tanned by $10 \mathrm{~g} / \mathrm{L}$ DSA (based on the volume of water, the same below) at $25^{\circ} \mathrm{C}$ for $4 \mathrm{~h}$ and basified with $12 \mathrm{~g} / \mathrm{L}$ sodium bicarbonate to $\mathrm{pH}$ 8.0. Then, the tanning temperature was raised to $40^{\circ} \mathrm{C}$ and continuously ran for another $4 \mathrm{~h}$ to obtain tanned sheep fur [1]. Gelatin, sodium chloride, sodium carbonate, acetone, urea, sodium pyrosulfite, sodium hydrosulfite and sodium bisulfite, were of analytical grade and purchased from Chengdu Kelong Chemical Co. Ltd. (Chengdu, China). Chrome tanning agent $\left(\mathrm{Cr}_{2} \mathrm{O}_{3}, 24 \%\right)$ was supplied by Brother Enterprises Holding Co., Ltd. (Haining, China). The other chemicals used in fur processing were of commercial grade. Chemicals used for the analysis of fur were of analytical grade.

\subsection{Tanning mechanism analysis of DSA}

The tanning mechanism of DSA was analyzed by investigating the effect of rinsing medium on the Ts of rinsed fur and the interactions between DSA and gelatin. Five pieces of DSA tanned fur were respectively added into $500 \mathrm{~mL}$ conical flasks containing $200 \mathrm{~mL}$ of distilled water, sodium carbonate solution $(\mathrm{pH}=9.5)$, urea solution (10 wt\%), sodium chloride solution (10 wt\%), and acetone aqueous solution $(1: 1, \mathrm{v} / \mathrm{v})$. After shaking at $30{ }^{\circ} \mathrm{C}$ for $12 \mathrm{~h}$, it stood overnight [19]. Then, the treated fur was washed with distilled water and sampled to determine its Ts.

Gelatin solution $(20 \mathrm{~g} / \mathrm{L})$ and DSA solution $(20 \mathrm{~g} / \mathrm{L})$ were mixed with equal volume. Then, this mixture was shaken at $30^{\circ} \mathrm{C}$ for $4 \mathrm{~h}$. Then, $\mathrm{pH}$ of the mixture was adjusted to 7.8-8.0 and continuously shook for another $4 \mathrm{~h}$ to obtain their reaction product. The UV-vis spectra of gelatin solution, DSA solution as well as their reaction product were recorded by UV/VIS spectrophotometer (UV-1200, Mapada, Shanghai, China). Besides, the FT-IR spectra of lyophilized gelatin, DSA, and their reaction product were recorded using an FT-IR spectrometer (Nicolet 6700, Thermo Scientific, USA). Samples were pressed as $\mathrm{KBr}$ pellet and measured in the range of 400$4000 \mathrm{~cm}^{-1}$ at room temperature, using 32 scans and a resolution of $4 \mathrm{~cm}^{-1}$.

In addition, the effect of groups modification on Ts of leather was also investigated to clarify the groups on collagen fiber involved in DSA tanning reaction. Pickled cattle hide was selected for this investigation. It was 
modified by de-pickling, blocking carboxyl, deamination, and removing guanidyl [20,21]. The detailed method was described in supplementary materials.

\subsection{Fatliquoring of DSA tanned fur}

DSA tanned fur was fatliquored according to the process presented in Table S1. The dosage of fatliquor was 6,10 , 15 , and $20 \mathrm{~g} / \mathrm{L}$, respectively. After fatliquoring, the fur was sampled for Ts test, and the fatliquoring wastewater was sampled for the analysis of fatliquor uptake rate.

\subsection{Interactions between $\mathrm{Cr}$ (III) and DSA}

Chrome tanning agent solution and DSA solution were mixed with equal volume to control the concentration of chrome tanning agent and DSA was $2 \mathrm{~g} / \mathrm{L}$ and $10 \mathrm{~g} / \mathrm{L}$, respectively. Then, this mixture was shaken at $25^{\circ} \mathrm{C}$ for $4 \mathrm{~h}$. The UV-vis spectra of chrome tanning agent solution, DSA solution as well as their fresh mixture and reacted mixture $(4 \mathrm{~h})$ were recorded by UV/VIS spectrophotometer (UV-1200, Mapada, Shanghai, China). Besides, the FT-IR spectra of dried DSA and the DSA$\mathrm{Cr}$ (III) complex were recorded using FT-IR spectrometer (Tensor 27, Bruker, Germany). Samples were pressed as $\mathrm{KBr}$ pellet and measured in the range of 400$4000 \mathrm{~cm}^{-1}$ at room temperature, using 32 scans and a resolution of $4 \mathrm{~cm}^{-1}$.

\subsection{Chrome-reduced combination tanning trials}

Chrome retanning of DSA tanned sheep fur was performed in a drum with $800 \%$ water (based on the weight of tanned sheep fur, similarly hereinafter). First, a certain amount of formic acid (10 wt\%) was added to adjust the $\mathrm{pH}$ of tanning liquor to 2.8-3.0, and then a certain amount of chrome tanning agent $(1,2,3,4 \mathrm{~g} / \mathrm{L}$, respectively) was added. After running at room temperature for $4 \mathrm{~h}$, the $\mathrm{pH}$ of tanning liquor was raised to $4.0-4.2$ by adding sodium bicarbonate. Then the temperature was increased to $40^{\circ} \mathrm{C}$. After continuously running for $4 \mathrm{~h}$, the chrome retanned sheep fur was washed for $5 \mathrm{~min}$ and piled for $24 \mathrm{~h}$ for further analysis. This tanning scheme was set as Scheme A. Other two tanning schemes were conducted for performance comparisons, which were defined as Scheme B and Scheme C, respectively. The detailed tanning processes were presented in Table S1 and Table S2. The combination tanned fur prepared from the optimal tanning scheme was dyed at $68^{\circ} \mathrm{C}$ according to the process reported by our previous work [4].

\subsection{Analytical methods}

\subsubsection{Shrinkage temperature and area yield of fur}

Shrinkage temperature (Ts) is commonly used to characterize the hydrothermal stability of leather/fur. The Ts of fur was measured and recorded using digital leather shrinkage temperature tester (MSW-YD4, Shaanxi University of Science and Technology, China). The rate of heating was set to $2^{\circ} \mathrm{C} / \mathrm{min}$. Pickled and tanned furs were wrung to control its moisture content around $65 \%$. Then, the area of fur was measured by using the grid counting method $\left(1 \mathrm{~cm}^{2} /(\right.$ grid unit)). The area yield of fur was the ratio between the area of tanned/post-tanned fur and pickled fur.

\subsubsection{Resistance to acid rinsing and washing}

$20 \mathrm{~g}$ DSA tanned fur and $160 \mathrm{~g}$ water were added into the drum and then ran at $30^{\circ} \mathrm{C}$ for $20 \mathrm{~min}$. Then, a certain amount of formic acid aqueous solution (10 wt\%) was added to adjust the float $\mathrm{pH}$ as 6.0, 5.0, 4.0, 3.0, and 2.0 , respectively, and continuously ran for $1,2,3,4 \mathrm{~h}$. After that, the Ts of rinsed fur was determined for the analysis of resistance to acid rinsing. DSA tanned fur was washed with distilled water at $40^{\circ} \mathrm{C}$ for $1,2,4,6,8$, $10,12 \mathrm{~h}$, respectively. The Ts of washed fur was tested for the analysis of resistance to washing.

\subsubsection{Resistance to reductants}

$20 \mathrm{~g}$ DSA tanned fur and $160 \mathrm{~g}$ water were added into the drum and ran for $5 \mathrm{~min}$ at room temperature, and then a certain amount $(0.5,1.0,1.5,2.0 \mathrm{wt} \%)$ of sodium pyrosulfite (SP), sodium hydrosulfite ( $\mathrm{SH}$ ) and sodium bisulfite $(\mathrm{SB})$ were respectively added into the drum. After that, the drum ran at $30^{\circ} \mathrm{C}$ for $60 \mathrm{~min}$, and then the treated fur was sampled to test its Ts for the analysis of resistance to reductants.

\subsubsection{UV-irradiation}

DSA tanned fur and conventional formaldehyde tanned fur were air-conditioned for $24 \mathrm{~h}$ according to standard methods. Then, the air-conditioned furs were irradiated using a UV light $(\lambda=365 \mathrm{~nm}, 8 \mathrm{~W})$ for $0,3,6,12,24 \mathrm{~h}$, respectively. The color parameters $(\mathrm{L}, \mathrm{a}, \mathrm{b})$ of irradiated furs were recorded using a color measurement instrument (Color reader CR-13, Konica Minolt, Japan). The total color difference $(\Delta \mathrm{E})$ was calculated by eq. (1).

$$
\Delta E=\sqrt{(\Delta L)^{2}+(\Delta a)^{2}+(\Delta b)^{2}}
$$

$\Delta \mathrm{E}$ represents the overall color difference; $\Delta \mathrm{L}$ is the lightness difference; $\Delta \mathrm{a}$ and $\Delta \mathrm{b}$ stand for the difference of a and b values. $\Delta \mathrm{L}, \Delta \mathrm{a}$ and $\Delta \mathrm{b}$ were calculated by subtracting the corresponding values for standard white $(\mathrm{L}=$ 93.8, $\mathrm{a}=-0.5, \mathrm{~b}=3.6$ ).

\subsubsection{Chrome content in sheep fur and wastewater}

The chrome content in sheep fur and wastewater was determined according to the method reported in our previous work [4]. Sheep fur was completely shaved and cut into small particles. After drying to a constant 
weight, the fur samples were digested using concentrated nitric acid and hydrogen peroxide $(2: 1, \mathrm{v} / \mathrm{v})$. The digestion liquor was filtered and then diluted with deionized water. The concentration of chrome was determined by ICP-OES (Optima 8000, PerkinElmer, USA). The chrome content of fur was calculated by eq. (2).

$$
\begin{aligned}
& \text { Chrome content in tanned fur } \\
& =\frac{\text { Weight of } \mathrm{Cr}_{2} \mathrm{O}_{3}}{\text { Weight of dried fur }} \times 100 \%
\end{aligned}
$$

$10 \mathrm{~mL}$ tanning or dyeing wastewater was digested using a certain amount of concentrated nitric acid and hydrogen peroxide $(2: 1, \mathrm{v} / \mathrm{v})$ at first, and then the chrome concentration was determined as above. The uptake rate of chrome was calculated by eq. (3).

$$
\text { Uptake rate of chrome }=\frac{\mathrm{Cr}_{0}-\mathrm{Cr}_{1}}{\mathrm{Cr}_{0}} \times 100 \%
$$

$\mathrm{Cr}_{0}$ : the initial additive amount of chrome; $\mathrm{Cr}_{1}$ : the concentration of chrome in tanning wastewater multiplied by the volume of tanning wastewater.

\subsubsection{Uptake rate of fatliquor}

The uptake rate of fatliquor was determined according to the method reported in our previous work [22]. The raw fatliquor solution and fatliquoring wastewater were diluted by a certain multiple, and then $200 \mu \mathrm{L}$ diluted solution was added into $\mathrm{COD}_{\mathrm{Cr}}$ reagent tube. After being heated at $150{ }^{\circ} \mathrm{C}$ for $2 \mathrm{~h}$, the $\mathrm{COD}_{\mathrm{Cr}}$ value was recorded using HI99109COD spectrophotometer (HANNA, Italy). The uptake rate of fatliquor was calculated according to eq. (4):

$$
\text { Uptake rate of fatliquor }=\frac{F_{0}-F_{1}}{F_{0}} \times 100 \%
$$

$\mathrm{F}_{0}$ : $\mathrm{COD}_{\mathrm{Cr}}$ value of diluted raw fatliquor solution multiplied by the dilution multiple; $\mathrm{F}_{1}: \mathrm{COD}_{\mathrm{Cr}}$ value of diluted fatliquoring wastewater multiplied by the dilution multiple.

\subsubsection{Physical properties}

Fatliquored fur with shaved wool was fist airconditioned for $48 \mathrm{~h}$ at $20^{\circ} \mathrm{C}$ and $65 \% \mathrm{RH}$, and then its tensile and tear strengths, as well as elongation at specified load $\left(5 \mathrm{~N} / \mathrm{mm}^{2}\right)$, were determined according to IUP test methods [23, 24]. The softness of fur was tested using a standard leather softness tester (GT-303, designed based on IUP standard [25], Gotech Testing Machines Inc., China) with a reading range from $0.1 \mathrm{~mm}$ to $10 \mathrm{~mm}$. The aperture was $20 \pm 0.1 \mathrm{~mm}$ for the measurement of softer apparel furs.

\section{Results and discussion}

\subsection{Tanning mechanism of DSA}

Fig. 1a shows the effect of rinsing medium on Ts of rinsed fur. After being rinsed with distilled water, there was no significant change in Ts, indicating that the combination of DSA and collagen fibers was relatively stable. After being rinsed with sodium chloride solution, the Ts of fur decreased by $0.3^{\circ} \mathrm{C}$, indicating that there was a small amount of ionic bonding between DSA and collagen fibers. After being rinsed with sodium carbonate solution, the Ts of fur did not change, implying that DSA and collagen fibers were mainly covalently bonded, and DSA tanned fur had strong resistance to alkali rinsing. After being rinsed with acetone and urea solutions, the Ts of fur decreased by $1.2^{\circ} \mathrm{C}$ and $2.2^{\circ} \mathrm{C}$, respectively, suggesting that there was hydrogen bonding between DSA and collagen fibers. Collagen molecules contain many carboxyl, amino and guanidyl groups, which can participate in tanning reaction. Fig. 1b illustrates the effect of groups modification on Ts of leather. After DSA tanning, the Ts of carboxyl-blocked leather increased by $21.6^{\circ} \mathrm{C}$ to $85.1{ }^{\circ} \mathrm{C}$, and the Ts of de-pickled leather without group modification increased by $20.7^{\circ} \mathrm{C}$ to $85.4{ }^{\circ} \mathrm{C}$. This shows that DSA exhibited tanning effect not by crosslinking the carboxyl groups of collagen molecules. However, the Ts of DSA tanned leather only increased by $3.5^{\circ} \mathrm{C}$ and $10.5{ }^{\circ} \mathrm{C}$ to $56.4{ }^{\circ} \mathrm{C}$ and $72.8^{\circ} \mathrm{C}$, respectively when the amino and guanidyl groups of collagen molecule were removed. The above results indicate that DSA mainly crosslinked with the amino groups on collagen molecules to form a covalent bond to exhibit tanning effect. Meanwhile, DSA could also form partial covalent crosslinks with the guanidyl group on the side chain on collagen molecule to enhance the tanning effect.

Fig. 1c shows the FT-IR spectra of gelatin, DSA, and DSA crosslinked gelatin. DSA has a symmetric vibration of aldehyde group around $1735 \mathrm{~cm}^{-1}$ [26], but it disappears on the spectrum of DSA crosslinked gelatin. This indicated that the aldehyde group of DSA had reacted with gelatin. As reported, the characteristic absorption peak of $\mathrm{C}=\mathrm{N}$ bond usually appears around $1623 \mathrm{~cm}^{-1}$ [27]. The absorption band around $1630 \mathrm{~cm}^{-1}$ is generally assigned to the amide I of gelatin [28]. However, after overlapping with the characteristic peak of gelatin amide bond at $1630 \mathrm{~cm}^{-1}$, there is a significantly enhanced absorption peak at $1626 \mathrm{~cm}^{-1}$. The appearance of this peak proves the existence of $\mathrm{C}=\mathrm{N}$ structure in DSA crosslinked gelatin. Moreover, the characteristic band of gelatin at $1543 \mathrm{~cm}^{-1}$ assigned to amide II is completely absent in the spectrum of crosslinked gelatin, proving the involvement of this group in the crosslinking reaction [26]. The results above indicated that the aldehyde groups on DSA and the amino groups on gelatin formed 

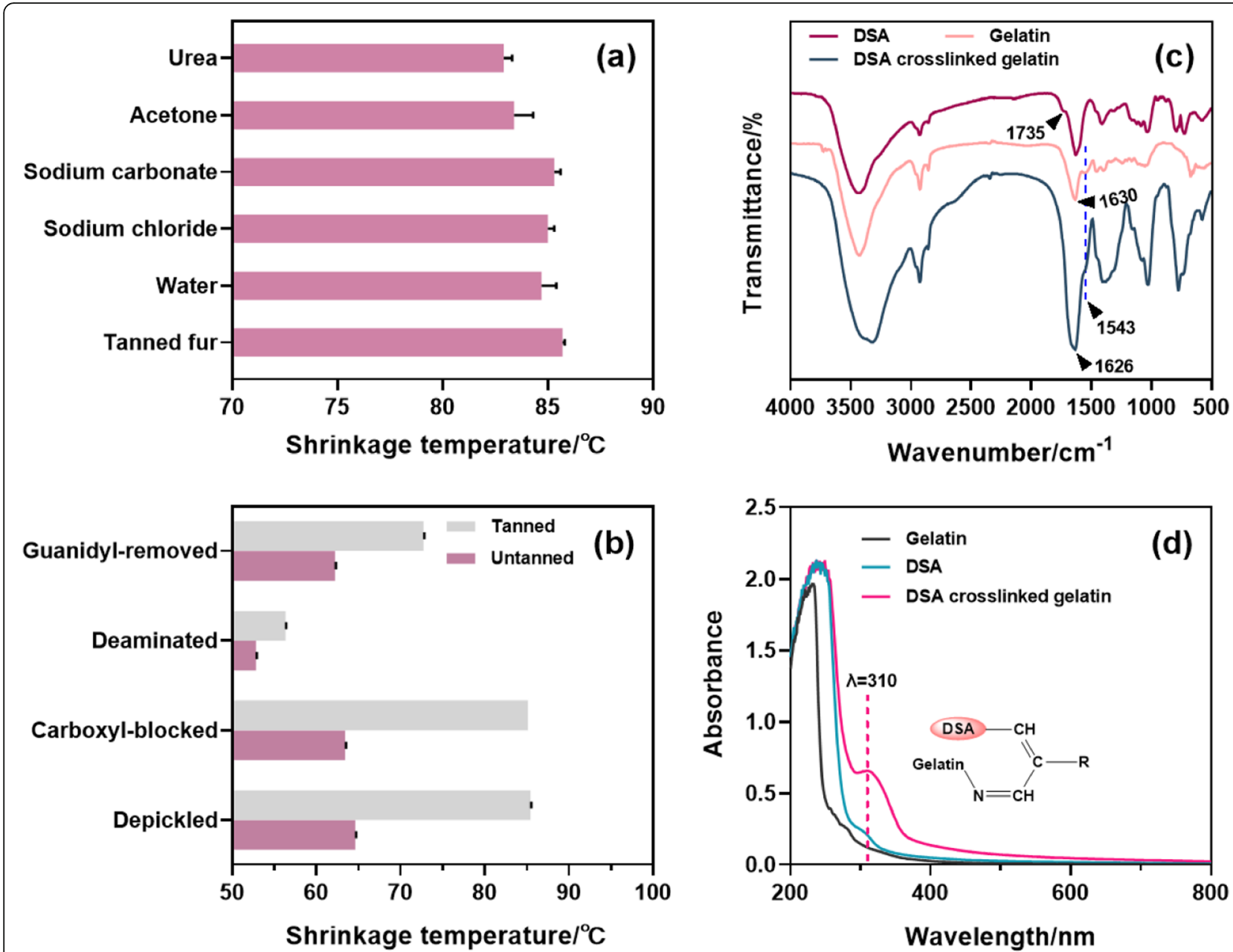

Fig. 1 Tanning mechanism analysis: a effect of rinsing medium on the Ts of rinsed fur; $\mathbf{b}$ effect of groups modification on the Ts of leather; $\mathbf{c}$ FTIR spectra of gelatin, DSA, and DSA crosslinked gelatin; d UV-vis spectra of gelatin, DSA, and DSA crosslinked gelatin solutions

Schiff base structures. Fig. 1d illustrates that a new absorption peak at $310 \mathrm{~nm}$ occurs in the spectrum of DSA crosslinked gelatin. This is because DSA may partially undergo $\beta$-elimination reaction to form $-\mathrm{C}=\mathrm{C}$ - structure under alkaline crosslinking conditions (around 8.0). The combination of $-\mathrm{C}=\mathrm{C}$ - structure with Schiff base structure may lead to the formation of a new conjugated structure $[29,30]$. These results demonstrated that DSA and gelatin did undergo Schiff base reactions to crosslink each other.

In summary, DSA was mainly combined with collagen fiber by forming Schiff base covalent bonds between aldehyde groups and amino/guanidyl groups while supplemented by a small number of hydrogen bonds and ionic bonds. The tanning mechanism of DSA was proposed and illustrated in Fig. 2, which was similar to the traditional aldehyde tanning mechanism [19]. Based on the tanning mechanism, the processing properties of DSA tanned sheep fur should be further investigated to guide the practical application of sustainable DSA tanning agent in the future.

\subsection{Stability of DSA tanned sheep fur \\ 3.2.1 Resistance to acid rinsing}

As is well acknowledged, the Schiff base is a dynamic covalent bond, which is less stable under acidic conditions than in basic conditions [31]. At present, the conventional post-tanning process of fur is generally conducted under acidic conditions. For instance, the fixation of dyestuff and fatliquor should be under an acidic condition with pH 3.8 to 4.0. Fig. 3 shows the Ts change of DSA tanned fur under different $\mathrm{pH}$ (a) and continuous washing (b). It can be found that the Ts of DSA tanned fur began to decrease when the float $\mathrm{pH}$ was lower than 6.0 , and it would decrease dramatically when the float $\mathrm{pH}$ was lower than 4.0. This indicated that the reversible reaction proceeded and resulted in the cleavage of Schiff base crosslinks between DSA and collagen fiber. 


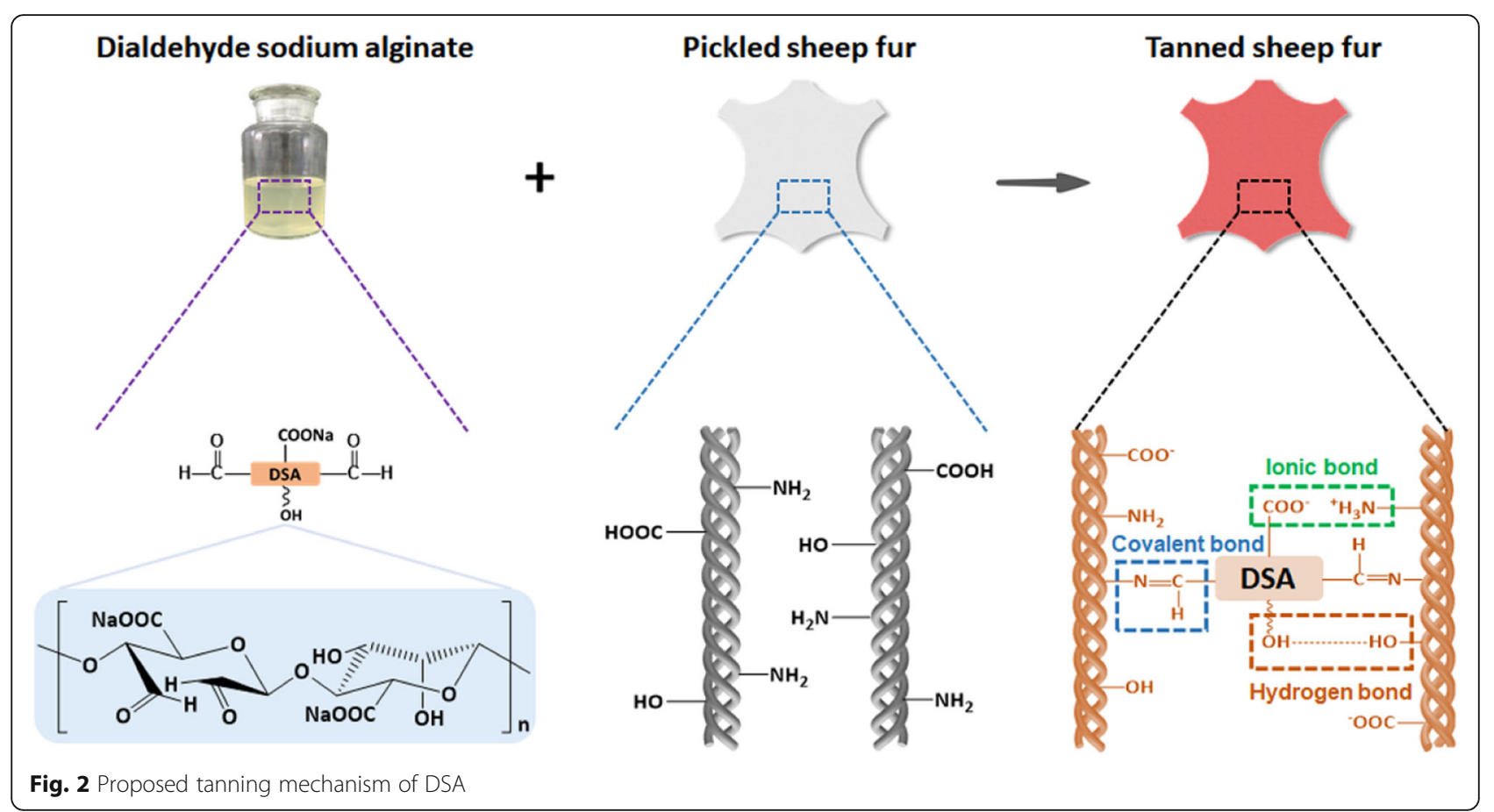

Whereas, the Ts of DSA tanned fur did not change significantly with the prolonging of duration under different acidic conditions. The above results indicate that the stability of Schiff base crosslinks between DSA and collagen fiber is dominated by the acidity of float. These crosslinks are relatively stable and not easily affected by the rinsing duration when the float acidity is fixed. It is worth noting that under the experimental conditions, the Ts of DSA tanned fur roughly remained at $82^{\circ} \mathrm{C}$ when the float $\mathrm{pH}$ decreased to 4.0, even though the rinsing duration was up to $4 \mathrm{~h}$. Fig. $3 \mathrm{~b}$ illustrates that the Ts of DSA tanned fur tended to be stable with prolonging the washing time. This indicated that the covalent crosslinks between the DSA and collagen fiber were relatively stable to washing, which was in agreement with the results shown in Fig. 1a. To sum up, DSA tanned fur
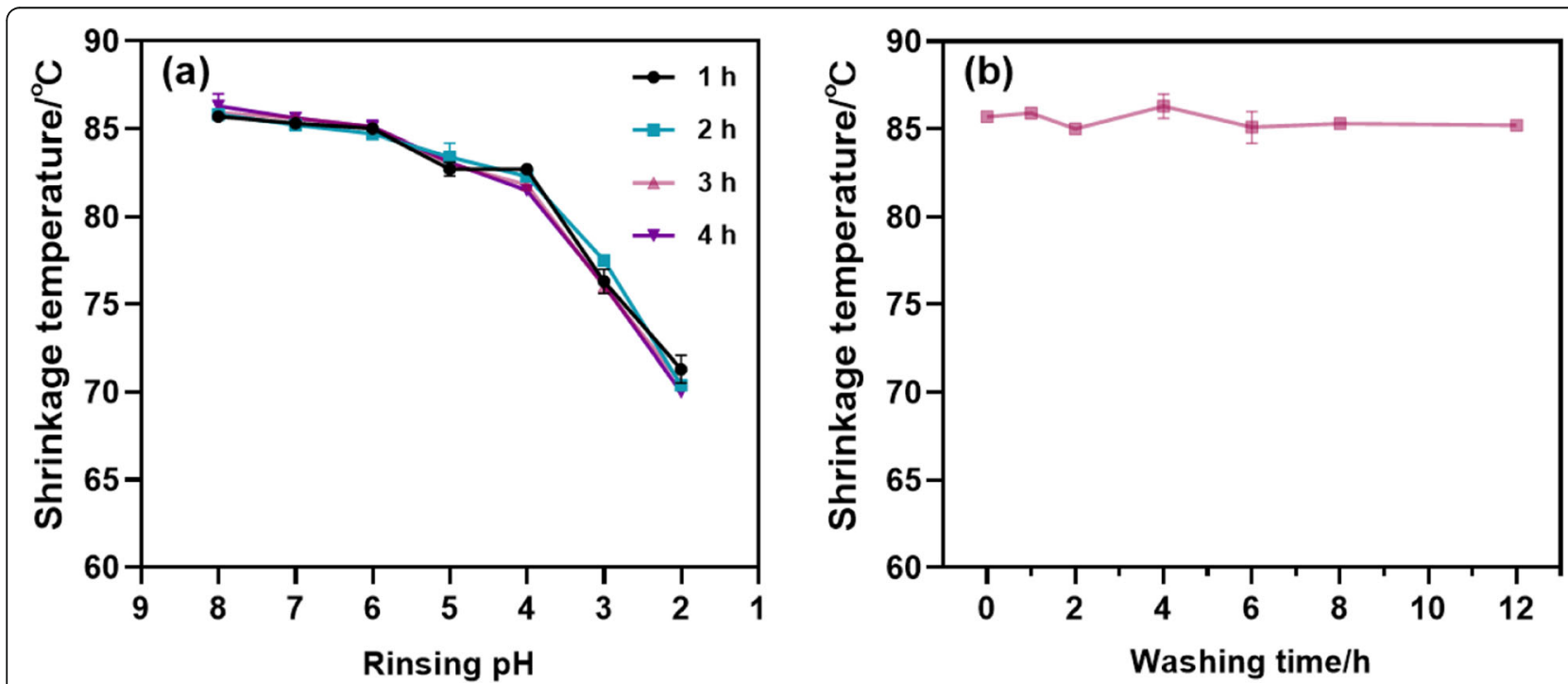

Fig. 3 Resistance of DSA tanned fur to rinsing: a the Ts change of DSA tanned fur under different $\mathrm{pH}$; $\mathbf{b}$ the Ts change of DSA tanned fur under continuous washing 
has undesirable resistance to acid but has favorable resistance to washing.

\subsubsection{Resistance to reductants}

In the post-processing of tanned fur, the fur may be bleached to remove the stains and pigment on the fur by some reductants. The effect of commonly used reductive bleaching agents, including sodium pyrosulfite (SP), sodium hydrosulfite $(\mathrm{SH})$ and sodium bisulfite $(\mathrm{SB})$, on the Ts of DSA tanned fur herein was investigated to evaluate the resistance to reductants. As can be seen from Fig. 4, the Ts of the tanned fur did not change obviously after the treatments with different reductants at a dosage of $0.5 \mathrm{wt} \%$. The Ts of treated fur tended to slightly decrease as the dosage of reductants continuously increased. When the dosage of reductant was up to $2 \mathrm{wt} \%$, the Ts of treated fur remained above $82^{\circ} \mathrm{C}$. According to the float $\mathrm{pH}$ presented in Fig. $4 \mathrm{~b}$, it can be inferred that the decrease of Ts might be due to the reversible reaction of Schiff base crosslinking caused by the increase of acidity of bleaching float. This shows that DSA tanned fur has good resistance to the commonly used reductive bleaching agents.

\subsubsection{Resistance to yellowing}

Resistance to yellowing is an important property for fur, which is generally evaluated according to the color change under UV irradiation. Thus, DSA tanned fur was treated by UV-irradiation to evaluate its resistance to yellowing. Formaldehyde (FA) tanned fur has a high resistance to yellowing, so it was treated in the same way for comparison. The $\Delta \mathrm{E}$ value of irradiated fur was calculated by subtracting the corresponding values for standard white to characterize the whiteness of irradiated fur, and lower $\Delta \mathrm{E}$ value generally means higher resistance to yellowing [17]. Moreover, the color change is slightly observable to human eyes when the $\Delta \mathrm{E}$ value is no higher than 1.5 [32]. Table 1 shows that the chromatic values of FA tanned fur had no obvious change, indicating that its whiteness was stable under UV irradiation and it had high resistance to yellowing. As illustrated in Fig. 5a, the $\Delta \mathrm{E}$ value of flesh side of DSA tanned fur was 2.24 when the UV irradiation time was prolonged to $12 \mathrm{~h}$, which would further increase as the irradiation time was continuously prolonged. However, it can be found from Table 1 that the $\mathrm{L}$ value of flesh side of DSA tanned fur increased and $\mathrm{b}$ value decreased as the irradiation time prolonged. This indicated that the flesh side of DSA tanned fur gradually became whiter under UV irradiation. Table 1 also shows that the L value of wool of DSA tanned fur slightly decreased and $\mathrm{b}$ value increased as the irradiation time prolonged. This implied that the wool of DSA tanned fur slightly yellowed under UV irradiation. However, the wool of DSA tanned fur had slightly higher $\Delta \mathrm{E}$ value compared with the wool of FA tanned fur (Fig. 5b). Overall, it can be deemed that DSA tanned fur has good resistance to yellowing.

\subsection{Fatliquoring performance}

Fatliquoring is an essential process for fur manufacturing to enable the resultant fur product with desirable softness. In general, the fatliquoring process of fur is conducted at $50{ }^{\circ} \mathrm{C}$, and the fixation of fatliquor was under the acidity with a $\mathrm{pH}$ around 4.0. Thus, in consideration of the acid-sensitivity and reversibility of Schiff


Fig. 4 Effect of reductants on the shrinkage temperature of DSA tanned fur: a effect of reductant dosage; $\mathbf{b}$ pH of solutions 
Table 1 Effect of UV-irradiation on the chrominance of tanned sheep fur

\begin{tabular}{|c|c|c|c|c|c|c|c|c|c|c|c|c|}
\hline \multirow{3}{*}{$\begin{array}{l}\text { Irradiation } \\
\text { time/h }\end{array}$} & \multicolumn{6}{|c|}{ Flesh side } & \multicolumn{6}{|c|}{ Wool } \\
\hline & \multicolumn{3}{|c|}{ DSA tanned fur } & \multicolumn{3}{|c|}{ FA tanned fur } & \multicolumn{3}{|c|}{ DSA tanned fur } & \multicolumn{3}{|c|}{ FA tanned fur } \\
\hline & $\mathrm{L}$ & $a$ & b & $\mathrm{L}$ & $\mathrm{a}$ & b & $\mathrm{L}$ & $\mathrm{a}$ & b & $\mathrm{L}$ & $\mathrm{a}$ & b \\
\hline 0 & 86.9 & 0.4 & 16.5 & 85.9 & 1.1 & 10.1 & 82.7 & 0.2 & 12.9 & 79.4 & 0.1 & 11.9 \\
\hline 3 & 86.8 & 0.8 & 15.7 & 85.3 & 1.4 & 10.4 & 82.2 & 0.6 & 13.8 & 78.6 & 0.5 & 11.9 \\
\hline 6 & 86.5 & 1.2 & 15.7 & 85.4 & 1.4 & 10.3 & 81.7 & 0.8 & 13.8 & 78.7 & 0.6 & 12.3 \\
\hline 12 & 87.1 & 1.1 & 14.4 & 85.7 & 1.4 & 10.4 & 81.5 & 1.1 & 13.5 & 78.5 & 0.7 & 11.8 \\
\hline 24 & 87.2 & 1.2 & 14.0 & 85.4 & 1.5 & 10.5 & 81.6 & 1.2 & 13.6 & 78.6 & 0.8 & 11.7 \\
\hline
\end{tabular}

base crosslinks, the fatliquoring performance of DSA tanned sheep fur was investigated. Fig. 6a illustrates that the Ts of fatliquored fur decreased slightly as the fatliquor concentration raised from $6 \mathrm{~g} / \mathrm{L}$ to $20 \mathrm{~g} / \mathrm{L}$. After fatliquoring, the Ts of fur decreased by $4.1{ }^{\circ} \mathrm{C}$ to $11.3{ }^{\circ} \mathrm{C}$ in comparison with the Ts of DSA tanned fur (Fig. 3b). This might be due to the cleavage of Schiff base crosslinks between DSA and collagen fiber under acidic conditions, which could be verified by the resistance to acid of DSA tanned fur (Fig. 3a). In addition, the fatliquor accumulated between the collagen fibers to increase the chain spacing between collagen molecules, thereby weakening the interaction degree between collagen fibers [33]. Higher dosage of fatliquor could result in a stronger weakening effect, thus the Ts of fatliquored tanned fur gradually decreased with the increase of fatliquor dosage.

DSA tanning will consume the amino groups on collagen fiber and introduce carboxyl groups onto the collagen fiber, thus reducing the positive charge of tanned fur [17]. This will be negative for the uptake and fixation of conventional fatliquor [31, 34]. As shown in Fig. 6b, the uptake rate of fatliquor for DSA tanned fur gradually increased with the increase of fatliquor concentration, which could reach to $85.4 \%$ when the fatliquor concentration was raised to $15 \mathrm{~g} / \mathrm{L}$. However, the uptake rate of fatliquor began to decrease with continuously increasing the dosage of fatliquor. This was mainly due to the limited capability of DSA tanned fur to absorb and combine fatliquor. Table 2 shows the physical properties of fatliquored DSA tanned fur after being air-dried, softened, and air-conditioned. The mechanical strengths, softness and extensibility of fatliquored fur gradually improved with the increase of fatliquor dosage. This was mainly because the surface of collagen fibers was covered by fatliquor to form a lubricating layer between collagen fibers, thus reducing the sliding friction between collagen fibers. Therefore, the softness, tear and tensile strengths of fatliquored fur were improved. As shown in Fig. 6a, the DSA tanned fur was severely de-tanned under these conditions, thereby greatly reducing the crosslinking density between collagen fibers. This performed negative








Fig. 6 Fatliquoring performance of DSA tanned fur: a effect of fatliquor dosage on the Ts of tanned fur; $\mathbf{b}$ Uptake rate of fatliquor

impacts on the above-mentioned properties of fatliquored fur. Thus, the physical properties of fatliquored fur began to decrease when the fatliquor concentration was higher than $15 \mathrm{~g} / \mathrm{L}$. The dosage of fatliquor needs to be reasonably controlled to obtain desirable resultant fur products when the DSA tanned fur undergoes the fatliquoring process. To sum up, DSA tanned fur has good fatliquoring performance when an appropriate amount of fatliquor is used, which can meet the requirements for making light-colored sheep fur products without high-temperature dyeing.

\subsection{Chrome-reduced combination tanning performance based on DSA}

3.4.1 Interactions between DSA and chrome tanning agent In practice, in addition to the aforementioned lightcolored sheep fur, the wool of sheep fur often needs to be dyed to manufacture dark-colored sheep fur products for meeting the market demands. Wool dyeing is generally carried out by using acid dyes in the temperature range of $65^{\circ} \mathrm{C}$ to $85^{\circ} \mathrm{C}$. Under such severe conditions, high hydrothermal stability of sheep fur is required to prevent the fur from shrinking, and our previous studies have shown that the Ts of fur generally needs to be higher than $90^{\circ} \mathrm{C}$ [4]. It can be seen that the Ts of DSA tanned sheep fur (around $85^{\circ} \mathrm{C}$ ) did not meet this requirement and should be further improved. Chromereduced combination tanning of sheep fur has been proven to be an alternative strategy [4, 35]. Considering that DSA contains multifunctional groups, e.g., carboxyl group, hydroxyl group and aldehyde group, it has the potential to interact with $\mathrm{Cr}$ (III) to form a more robust crosslinking network in the collagen fiber matrix of fur. Fig. 7a shows the UV-vis spectra of the complex of DSA and $\mathrm{Cr}$ (III). The fresh chrome tanning agent solution (2 $\mathrm{g} / \mathrm{L}$ ) had two absorption peaks assigned to $\mathrm{Cr}$ (III) at $\lambda_{1}-$ $430 \mathrm{~nm}$ and $\lambda_{2}-595 \mathrm{~nm}$, and DSA $(10 \mathrm{~g} / \mathrm{L})$ had no absorption peaks here. In the fresh mixture of chrome tanning agent and DSA, the two absorption peaks of $\mathrm{Cr}$ (III) shifted to $\lambda_{1}-420 \mathrm{~nm}$ and $\lambda_{2}-592 \mathrm{~nm}$, respectively. This indicated that the DSA molecule was coordinated with $\mathrm{Cr}$ (III). After reaction for $4 \mathrm{~h}$, the two absorption peaks of $\mathrm{Cr}$ (III) continuously shifted to $\lambda_{1}-408 \mathrm{~nm}$ and $\lambda_{2}-575 \mathrm{~nm}$, respectively. This suggested that $\mathrm{Cr}$ (III) was further coordinated with the carboxyl and/or hydroxyl groups on DSA molecules. The blue shift of the two absorption peaks demonstrates that there was an obvious steric hindrance effect of the DSA-Cr (III) complex [36],

Table 2 Physical properties of fatliquored DSA tanned sheep fur

\begin{tabular}{lllll}
\hline Dosage of fatliquor/(g/L) & Tensile strength/(N/mm $\mathbf{2})$ & Tear strength/(N/mm) & Elongation at a load of $\mathbf{5} \mathbf{~ N} / \%$ & Softness/mm \\
\hline 6 & $15.93 \pm 0.54$ & $38.32 \pm 5.60$ & $33.14 \pm 1.76$ & $8.02 \pm 0.30$ \\
10 & $17.79 \pm 1.32$ & $49.91 \pm 4.51$ & $36.24 \pm 8.96$ & $8.11 \pm 0.24$ \\
15 & $22.12 \pm 2.73$ & $62.77 \pm 10.05$ & $42.50 \pm 6.99$ & $8.15 \pm 0.13$ \\
20 & $15.89 \pm 0.26$ & $42.62 \pm 1.84$ & $31.14 \pm 7.53$ & $8.13 \pm 0.45$ \\
\hline
\end{tabular}



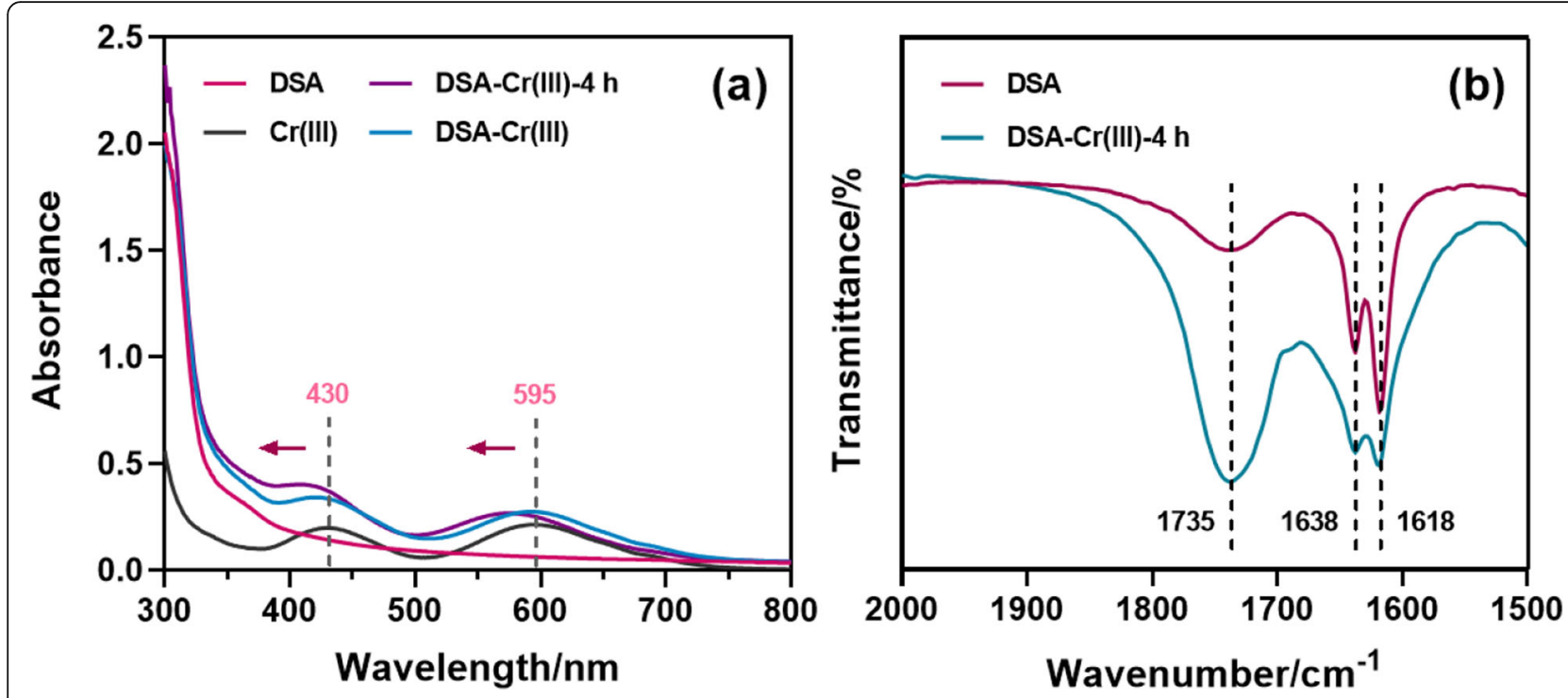

Fig. 7 UV-vis (a) and FT-IR (b) spectra of DSA, $\mathrm{Cr}$ (III) and the complex of DSA and $\mathrm{Cr}$ (III)

which might be caused by the macromolecular characteristics of DSA. Moreover, the ratio of absorbances at $\lambda_{1}-408 \mathrm{~nm}(0.4014)$ and $\lambda_{2}-575 \mathrm{~nm}(0.2659)$ was 1.51 , indicating that DSA was coordinated with Cr (III) in terms of bridge bonding or cyclic coordination bonding [37].

The FT-IR spectra of the complex of DSA and Cr (III) is shown in Fig. 7b. The absorption bands at $1638 \mathrm{~cm}^{-1}$ and $1618 \mathrm{~cm}^{-1}$ were assigned to the antisymmetric stretching and symmetrical vibrations of carboxylate ions on DSA. The absorption band at $1735 \mathrm{~cm}^{-1}$ was assigned to symmetric vibration of aldehyde group. After coordinating with $\mathrm{Cr}$ (III), the vibration intensity of the absorption band assigned to carboxylate ion was obviously decreased. The new absorption band assigned to the carboxylate combined with $\mathrm{Cr}$ (III) was overlapped by the absorption band assigned to the carboxylate on excessive DSA. Whereas, it can be also inferred that the difference between the antisymmetric and symmetrical vibration frequencies of carboxylate was lower than 100 $\mathrm{cm}^{-1}$, indicating DSA and Cr (III) might be coordinated in a chelated form [37]. The above results demonstrate that DSA can be complexed with $\mathrm{Cr}$ (III), providing the feasibility of chrome-reduced combination tanning of sheep fur based on DSA.

\subsubsection{General tanning performance}

According to the interactions between DSA and Cr (III), DSA tanning of sheep fur combined with reduced chrome tanning agent was proposed. As illustrated in Fig. 8, three tanning schemes were designed, including "DSA tanning first and then chrome retanning" (Scheme A), "DSA and chrome tanning in the same bath"
(Scheme B), and "chrome tanning first and then DSA retanning" (Scheme C). The Ts of fur tanned by Scheme A was improved to $90.2^{\circ} \mathrm{C}$, which met the Ts requirement of high-temperature dyeing. When the fur was tanned by Scheme B, the Ts of tanned fur was only $68.2^{\circ} \mathrm{C}$, which was $10.7^{\circ} \mathrm{C}$ lower than that of fur only tanned by $2 \mathrm{~g} / \mathrm{L}$ chrome tanning agent $\left(78.9^{\circ} \mathrm{C}\right)$ [38]. The Ts of fur tanned by Scheme $\mathrm{C}$ was $85.6^{\circ} \mathrm{C}$, which was not further improved and comparable to that of fur only tanned by $10 \mathrm{~g} / \mathrm{L}$ DSA $\left(85.7^{\circ} \mathrm{C}\right)$. Fig. $8 \mathrm{~b}$ shows that the uptake rate of chrome tanning agent and the $\mathrm{Cr}$ content of fur tanned by different schemes were rather different. Our previous work showed that the $\mathrm{Cr}$ content of sheep fur tanned only by $2 \mathrm{~g} / \mathrm{L}$ chrome tanning agent was $0.73 \%$ (calculated as $\mathrm{Cr}_{2} \mathrm{O}_{3}$, similarly hereinafter) [38]. The Cr content of fur tanned by Scheme A was $0.96 \%$, which was obviously higher than that of fur tanned by the same dosage of chrome tanning agent. This shows that DSA tanning could promote the subsequent penetration and fixation of chrome tanning agent in the collagen fiber matrix of fur. When tanning Scheme B was employed, the $\mathrm{Cr}$ content of tanned fur was the lowest (only 0.47\%), which was significantly lower than that of fur only tanned by $2 \mathrm{~g} / \mathrm{L}$ chrome tanning agent. This indicates that DSA will prevent the penetration of chrome tanning agent into the collagen fiber matrix of fur, thus DSA and chrome tanning agent cannot be used in the same tanning bath. When the fur was tanned using Scheme $\mathrm{C}$, the Cr content of tanned fur was $0.69 \%$, which was slightly lower than that of fur only tanned by $2 \mathrm{~g} / \mathrm{L}$ chrome tanning agent. This might be attributed to the release of small amount of chrome from tanned fur during the later DSA tanning process. 

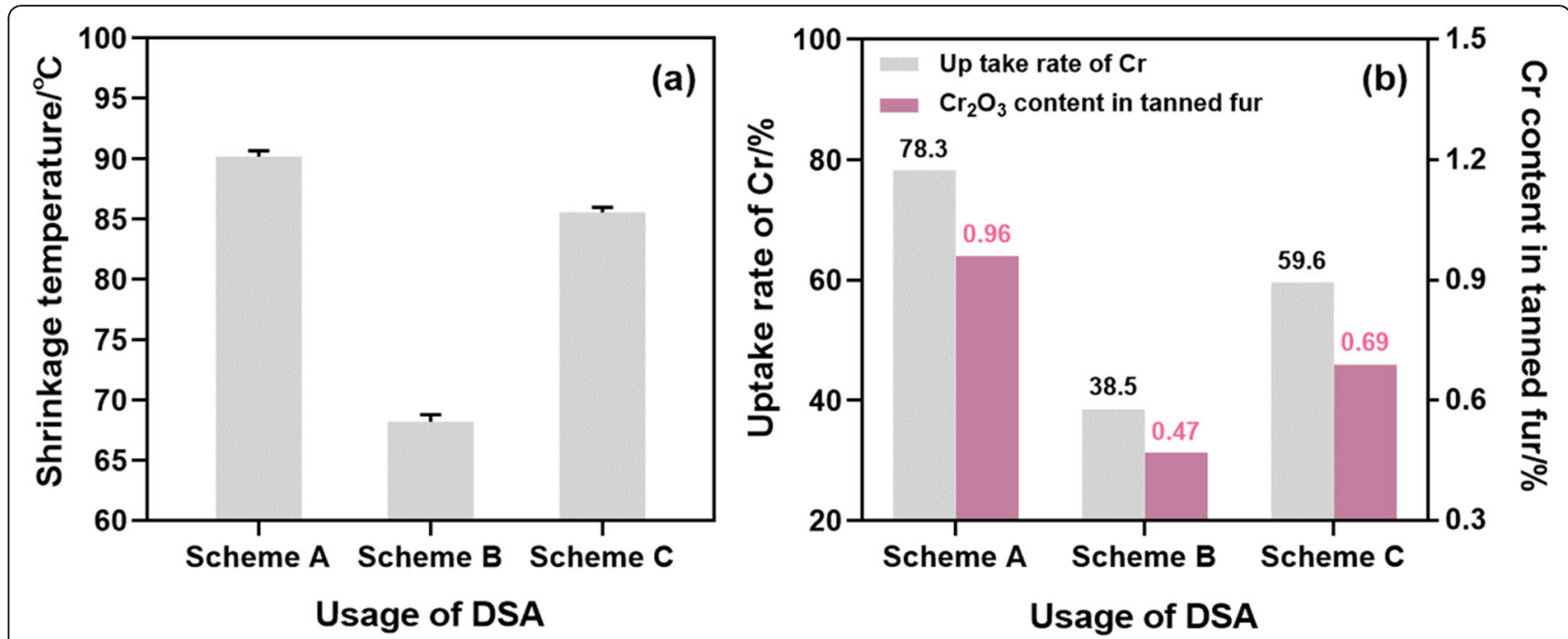

Fig. 8 Effect of the DSA usage (tanning scheme) on the Ts of tanned fur (a) and the uptake of $\mathrm{Cr}$ by fur (b); the dosage of DSA was $10 \mathrm{~g} / \mathrm{L}$, the dosage of chrome tanning agent was $2 \mathrm{~g} / \mathrm{L}$

The reason for the difference in tanning performances of the three tanning schemes can be explained by the strong binding interaction between DSA and Cr (III). Since Cr (III) had been fully coordinated with the carboxyl groups on collagen fiber, few $\mathrm{Cr}$ (III) could combine with the carboxyl groups on DSA molecules to further strengthen the tanning effect. Compared with fur only tanned by DSA, the Ts of fur tanned by Scheme A had no obvious increase, and tanning Scheme $C$ should not be suggested. Based on the results presented in Fig. 7, Cr (III) could form stable coordination bonds with the carboxyl and/or hydroxyl groups on macromolecular DSA, making DSA molecules become larger. Thus, the DSA$\mathrm{Cr}$ (III) complex was difficult to penetrate into the collagen fiber matrix of fur. Meanwhile, Cr (III) also lost partial capability to coordinate with carboxyl groups on collagen fiber, so the Ts of tanned fur was the lowest, and tanning Scheme B should not be adopted. As for tanning Scheme A, DSA was fixed in the collagen fiber matrix of fur in advance by forming Schiff base crosslinks, resulting in the dispersion of collagen fibers to expand the penetration channel for subsequent chrome tanning agent. When $\mathrm{Cr}$ (III) penetrated into the collagen fiber matrix of fur, it could not only combine with the carboxyl groups on collagen fiber, but also coordinate with the carboxyl groups on DSA molecules. Finally, the absorption and fixation of Cr (III) was improved, thereby increasing the effective crosslinking between collagen fibers and improving the Ts of tanned fur. As a result, the tanning Scheme A endowed tanned fur with the highest Ts, thus it should be recommended for the production of dark-colored sheep fur.

\subsubsection{High-temperature dyeing performance and physical properties}

The effects of high-temperature dyeing on $\mathrm{Cr}$ release, Ts, and area yield of the combination tanned fur were investigated. As shown in Table 3, the released $\mathrm{Cr}$ from the fur during high-temperature dyeing was lower than the discharge standard of total $\mathrm{Cr}$ in tannery wastewater from workshop outlet $(1.5 \mathrm{mg} / \mathrm{L}$, in China) [1], which was less than that from the conventional chrome tanned fur under the same offer of chrome tanning agent [4]. This means that the combination tanning using DSA enhanced the fixation of $\mathrm{Cr}$ since more carboxyl groups were introduced onto collagen fibers. Table 3 also shows

Table 3 High-temperature dyeing performance of chrome-reduced combination tanned sheep fur

\begin{tabular}{|c|c|c|c|c|c|}
\hline \multirow{2}{*}{$\begin{array}{l}\text { Tanning } \\
\text { scheme }\end{array}$} & \multirow{2}{*}{$\begin{array}{l}\text { Cr conc. in } \\
\text { dyeing } \\
\text { wastewater/ } \\
\text { (mg/L) }\end{array}$} & \multicolumn{2}{|l|}{$\mathrm{Ts} /{ }^{\circ} \mathrm{C}$} & \multicolumn{2}{|l|}{ Area yield/\% } \\
\hline & & Before dyeing & After dyeing & Before dyeing & After dyeing \\
\hline$\overline{D S A}+1 C r^{a}$ & $0.88 \pm 0.01$ & $86.0 \pm 0.5$ & $80.0 \pm 1.2$ & $119.9 \pm 0.5$ & $107.5 \pm 0.0$ \\
\hline $\mathrm{DSA}+2 \mathrm{Cr}$ & $1.18 \pm 0.01$ & $91.0 \pm 0.3$ & $83.1 \pm 1.1$ & $131.4 \pm 0.0$ & $131.4 \pm 0.0$ \\
\hline $\mathrm{DSA}+3 \mathrm{Cr}$ & $1.24 \pm 0.02$ & $93.6 \pm 0.3$ & $88.7 \pm 0.2$ & $125.4 \pm 0.0$ & $125.4 \pm 0.0$ \\
\hline $\mathrm{DSA}+4 \mathrm{Cr}$ & $1.46 \pm 0.01$ & $97.2 \pm 0.1$ & $89.8 \pm 0.9$ & $117.1 \pm 0.0$ & $117.1 \pm 0.0$ \\
\hline
\end{tabular}

aickled sheep fur was tanned by $10 \mathrm{~g} / \mathrm{L}$ DSA and then retanned by $2 \mathrm{~g} / \mathrm{L}$ chrome tanning agent, similarly hereinafter 
Table 4 Physical properties of fatliquored sheep fur made from chrome-reduced combination tanning based on DSA

\begin{tabular}{|c|c|c|c|c|}
\hline Tanning scheme & Tensile strength/(N/mm $\left.{ }^{2}\right)$ & Tear strength/(N/mm) & Elongation at a load of $5 \mathrm{~N} / \%$ & Softness $/ \mathrm{mm}$ \\
\hline$\overline{D S A}+1 \mathrm{Cr}$ & $11.06 \pm 2.70$ & $39.20 \pm 3.37$ & $22.83 \pm 2.94$ & $6.76 \pm 0.18$ \\
\hline $\mathrm{DSA}+2 \mathrm{Cr}$ & $16.27 \pm 2.61$ & $54.19 \pm 5.15$ & $23.58 \pm 1.97$ & $7.44 \pm 0.10$ \\
\hline $\mathrm{DSA}+3 \mathrm{Cr}$ & $17.86 \pm 0.07$ & $58.37 \pm 12.65$ & $24.46 \pm 0.08$ & $7.94 \pm 0.18$ \\
\hline $\mathrm{DSA}+4 \mathrm{Cr}$ & $18.00 \pm 0.17$ & $62.77 \pm 2.98$ & $25.90 \pm 2.12$ & $8.28 \pm 0.14$ \\
\hline
\end{tabular}

that the Ts decreased after high-temperature dyeing. This might be due to the partial cleavage of Schiff base crosslinks between DSA and collagen fiber under severe acidic conditions (around $\mathrm{pH} 4.0,50^{\circ} \mathrm{C}$ ). Even so, the area yields of the fur did not decrease except the fur tanned by $10 \mathrm{~g} / \mathrm{L}$ DSA and $1 \mathrm{~g} / \mathrm{L}$ chrome tanning agent. These results suggest that the chrome-reduced combination tanned fur based on DSA is capable of enduring the high-temperature dyeing process.

Physical properties of resultant fur determine its processing performance for fur products manufacturing. As our previous work reported, the tensile and tear strengths of fur made by conventional chrome tanning using $16 \mathrm{~g} / \mathrm{L}$ chrome tanning agent were $13.27 \mathrm{~N} / \mathrm{mm}^{2}$ and $49.67 \mathrm{~N} / \mathrm{mm}$, respectively [4]. Table 4 shows that mechanical strengths of the combination tanned dyed fur were higher than those of conventional chrome tanned fur when the chrome tanning agent used in the combination tanning was no lower than $2 \mathrm{~g} / \mathrm{L}$. The elongation at a specified load $(5 \mathrm{~N})$ and softness of resultant fur increased as the concentration of chrome tanning agent was raised. This was mainly due to the improved uptake of post-tanning chemicals [4]. Based on the results above, chrome-reduced retanning of DSA tanned sheep fur can be proposed as a feasible strategy to make dark-colored fur products that need hightemperature dyeing.

\section{Conclusions}

Sustainable dialdehyde sodium alginate (DSA) can be used as an organic tanning agent for the tanning of sheep fur. DSA was mainly combined with collagen fiber by forming Schiff base covalent bonds between the aldehyde groups and amino/guanidyl groups while supplemented by a small number of hydrogen bonds and ionic bonds. As a new tanned sheep fur, DSA tanned fur had poor resistance to acid rinsing due to the acid sensitivity of Schiff base structure, but it had excellent resistance to washing and good fatliquoring performance as well as good resistances to yellowing and reductant. Considering that macromolecular DSA can highly coordinate with $\mathrm{Cr}$ (III), DSA and chrome tanning agent cannot be used in the same tanning bath. Tanning of sheep fur using DSA first and then retanning by chrome tanning agent is the optimal combination tanning scheme, which can make the tanned fur meet the needs of high-temperature dyeing processing. The use of DSA brings about high fixation of chrome in the collagen fiber matrix of fur, thus this strategy can not only largely reduce the dosage of chrome tanning agent, but also produce dark-colored fur with favorable area yield and physical properties. Overall, the sheep fur tanned by sustainable DSA has favorable properties under appropriate post-tanning processing conditions to produce satisfactory resultant light-colored or dark-colored fur.

\section{Supplementary Information}

The online version contains supplementary material available at https://doi. org/10.1186/s42825-020-00047-8.

Additional file 1: Table S1. Fatliquoring process of DSA tanned fur;

Table S2. Process for DSA and chrome tanning in the same bath

(Tanning Scheme B); Table S3. Process for chrome-reduced tanning and DSA retanning (Tanning Scheme $\mathrm{C}$ ).

\section{Acknowledgments \\ This work was financially supported by the National Key R\&D Program (2017YFB0308500).}

\section{Authors' contributions}

WD designed and performed all the experiments, and drafted the manuscript. YW provided suggestions for the experiments and analyzed the results. JZ provided suggestions for the design of tanning trials. HL prepared the samples for FT-IR analysis and conducted the FT-IR determinations. XP provided resources for the FT-IR determinations. BS supervised the project and reviewed the manuscript. The authors read and approved the final manuscript.

\section{Availability of data and materials}

All data generated or analyzed during this study are included in this manuscript and the additional file. The authors declare that the data in this article is reliable.

\section{Competing interests}

The authors declare that they have no competing interests.

\section{Author details \\ ${ }^{1}$ China Leather and Footwear Research Institute Co. Ltd., Beijing 100015, P.R. China. ${ }^{2}$ College of Biomass Science and Engineering, Sichuan University, Chengdu 610065, China. ${ }^{3}$ Key Laboratory of Leather Chemistry and Engineering of Ministry of Education, Sichuan University, Chengdu 610065, P.R. China.}

Received: 17 September 2020 Accepted: 22 December 2020 Published online: 15 March 2021

\section{References}

1. Ding W, Yi Y, Wang Y, Zhou J, Shi B. Preparation of a highly effective organic tanning agent with wide molecular weight distribution from biorenewable sodium alginate. ChemistrySelect. 2018;3(43):12330-5. 
2. Huang $W$, Song $Y, Y u$ Y, Wang $Y$, Shi B. Interaction between retanning agents and wet white tanned by a novel bimetal complex tanning agent. J Leather Sci Eng. 2020;2:1-9.

3. $Y u$ Y, Zeng $Y$, Wang $Y$, Liang $T$, Zhou J, Shi B. Inverse chrome tanning technology: a practical approach to minimizing $\mathrm{Cr}$ (III) discharge. J Am Leather Chem Assoc. 2020;115(5):176-83.

4. $\quad$ Ding W, Cheng Y, Wang Y, Shi B. Chrome-reduced combination tanning for cleaner dyed sheep fur processing. J Am Leather Chem Assoc. 2015;110(11): 363-71.

5. Ding W, Wang Y, Zhou J, Shi B. Effect of structure features of polysaccharides on properties of dialdehyde polysaccharide tanning agent. Carbohydr Polym. 2018;201:549-56.

6. Ding W, Pang X, Ding Z, Tsang DCW, Jiang Z, Shi B. Constructing a robust chrome-free leather tanned by biomass-derived polyaldehyde via crosslinking with chitosan derivatives. J Hazard Mater. 2020;396:Article 122771

7. Yi Y, Jiang Z, Yang S, Ding W, Wang Y, Shi B. Formaldehyde formation during the preparation of dialdehyde carboxymethyl cellulose tanning agent. Carbohydr Polym. 2020;239:Article 116217

8. Xu W, Liu K, Li T, Zhang W, Dong Y, Lv J, et al. An in situ hydrogel based on carboxymethyl chitosan and sodium alginate dialdehyde for corneal wound healing after alkali burn. J Biomed Mater Res Part A. 2019;107(4):742-54.

9. Balakrishnan B, Jayakrishnan A. Self-cross-linking biopolymers as injectable in situ forming biodegradable scaffolds. Biomaterials. 2005;26(18):3941-51.

10. Gómez-Ordóñez E, Rupérez P. FTIR-ATR spectroscopy as a tool for polysaccharide identification in edible brown and red seaweeds. Food Hydrocoll. 2011;25(6):1514-20.

11. Emami Z, Ehsani M, Zandi M, Foudazi R. Controlling alginate oxidation conditions for making alginate-gelatin hydrogels. Carbohydr Polym. 2018; 198:509-17.

12. Gomez CG, Rinaudo M, Villar MA. Oxidation of sodium alginate and characterization of the oxidized derivatives. Carbohydr Polym. 2007;67(3): 296-304.

13. Gao C, Liu M, Chen J, Zhang X. Preparation and controlled degradation of oxidized sodium alginate hydrogel. Polym Degrad Stab. 2009;94(9):1405-10.

14. Boanini E, Rubini K, Panzavolta S, Bigi A. Chemico-physical characterization of gelatin films modified with oxidized alginate. Acta Biomater. 2010;6(2):383-8.

15. Ding W, Zhou J, Zeng $Y$, nan WY, Shi B. Preparation of oxidized sodium alginate with different molecular weights and its application for crosslinking collagen fiber. Carbohydr Polym. 2017;157:1650-6.

16. Hu Y, Liu L, Gu Z, Dan W, Dan N, Yu X. Modification of collagen with a natural derived cross-linker, alginate dialdehyde. Carbohydr Polym. 2014; 102:324-32

17. Ding W, Yi Y, Wang Y, Zhou J, Shi B. Peroxide-periodate co-modification of carboxymethylcellulose to prepare polysaccharide-based tanning agent with high solid content. Carbohydr Polym. 2019:224:Article 115169.

18. Cheng F, Zhang D, Wang X. Theory and technology of fur processing Beijing: Chemical Industry Press; 2005.

19. Qian Y, Liu W, Zhao S, Qiang X. Study on the performance and application of an organic phosphonium tanning agent. China Leather. 2006;35(19):19-22

20. Zhang L. Specificity of the modification reaction on collagen. J Beijing Institure Cloth Technol. 2004;24(4):32-6.

21. Ma J, Yang Z, Lu D, Feng L, Hu G. Study on the interaction between vinyl polymer tanning agents and skin collagen. China Leather. 2000;29(9):13-8.

22. Ding W, Wang Y, Zhou J, Li J, Shi B. Application of oxidized sodium alginate in chrome-free tanning process of cattle hide. China Leather. 2018:47(5):20-2 30.

23. IUP 6. Measurement of tensile strength and percentage elongation. J Soc Leather Technol Chem. 2000;84:317-21.

24. IUP 8. Measurement of tear load-double edge tear. J Soc Leather Technol Chem. 2000;84:327-9.

25. IUP 36. Measurement of leather softness. J Soc Leath Tech Ch. 2000:84: 377-9.

26. Sarker B, Papageorgiou DG, Silva R, Zehnder T, Gul-E-Noor F, Bertmer M, et al. Fabrication of alginate-gelatin crosslinked hydrogel microcapsules and evaluation of the microstructure and physico-chemical properties. J Mater Chem B. 2014;2(11):1470-82.

27. Ye J, Xiong J, Sun R. The fluorescence property of Schiff's bases of carboxymethyl cellulose. Carbohydr Polym. 2012;88(4):1420-4.
28. Aewsiri T, Benjakul S, Visessanguan W, Eun JB, Wierenga PA, Gruppen H. Antioxidative activity and emulsifying properties of cuttlefish skin gelatin modified by oxidised phenolic compounds. Food Chem. 2009;117(1):160-8.

29. Whistler RL, Chang PK, Richards GN. Alkaline degradation of periodateoxidized starch1. J Am Chem Soc. 1959;81(12):3133-6.

30. Whistler RL, Chang PK, Richards GN. Alkaline degradation of periodateoxidized xylan and dextran1. J Am Chem Soc. 1958;81(15):4058-60.

31. Ding $W$, Wu Y. Sustainable dialdehyde polysaccharides as versatile building blocks for fabricating functional materials: An overview. Carbohydr Polym. 2020;248:Article 116801.

32. Niu M, Dai J, Hou W, Zhang R. The effect of ultraviolet (UV) irradiation on the structures and properties of wool fiber. Mater Rev. 2010;24(14):33-6.

33. Yu K, Zhang F, Chen Z, Luo P, Xin Z. Study on the fatliquoring technology of sulfonated phospholipid fatliquor. Leather Sci Eng. 2009;19(3):55-9.

34. Wang Y, Shi B. Progress of inverse chrome tanning technology. Chem Ind Eng Prog. 2019;38(1):639-48.

35. Ding W, Wang Y, Li J, Zhou J, Ma J, Shi B. Optimization of organic tanning agents used for less chrome combination tanning of dyed sheep fur. China Leather. 2016;45(6):55-61,74.

36. Deng Y, Zhao A, Yin L, Qiu M, Jia W. Synthesis and characterization of baicalin-chromium (III) complex. Chin J Nat Med. 2007;5(1):38-41.

37. Ding W, Cheng $X$, Wang $W$, Zhang $W$. Theoretical study on the stability of coordination state of Cr (III) with carboxyl. Chem Res Appl. 2012;24(2):242-8.

38. Ding W, Zhang X, Wang Y, Shi B, Cao M. Chrome-reduced tanning for cleaner dyed sheep skin processing. In: 10th Asian International Conference on Leather Science and Technology; 2014. p. 46.

\section{Publisher's Note}

Springer Nature remains neutral with regard to jurisdictional claims in published maps and institutional affiliations.

\section{Submit your manuscript to a SpringerOpen ${ }^{\circ}$ journal and benefit from:}

- Convenient online submission

- Rigorous peer review

- Open access: articles freely available online

- High visibility within the field

- Retaining the copyright to your article

Submit your next manuscript at $>$ springeropen.com 